\title{
Sensitivity of ultrasound in detecting hepatocellular carcinoma in obese patients compared to explant pathology as the gold standard
}

\author{
Jamak Modaresi Esfeh ${ }^{1}$, Kaveh Hajifathalian ${ }^{2}$, and Kianoush Ansari-Gilani ${ }^{3}$ \\ 'Department of Gastroenterology and Transplant Hepatology, Cleveland Clinic, Cleveland, $\mathrm{OH}$; ${ }^{2}$ Department of Gastroenterology and \\ Transplant Hepatology, Weill Cornell Medicine, New York, NY; ${ }^{3}$ Department of Radiology, University Hospitals Cleveland Medical \\ Center, Cleveland, OH, USA
}

Background/Aims: The American Association for the Study of Liver Diseases recommends ultrasound (US) screening for hepatocellular carcinoma (HCC) among cirrhotic patients, regardless of body mass index (BMI), every 6 months. We examined US sensitivity for diagnosis of HCC in obese patients.

Methods: Liver transplant patients data with HCC in explant was used (January 2012-December 2017). All patients underwent liver US within 3 months of diagnosis of HCC. Number/size of HCC lesions were extracted from radiologic and pathologic reports. Obesity was defined as $\mathrm{BMI} \geq 30 \mathrm{~kg} / \mathrm{m}^{2}$.

Results: One hundred sixteen patients were included. $80 \%$ were male, with mean BMI of $31 \mathrm{~kg} / \mathrm{m}^{2}$. The most common underlying liver disease was hepatitis C virus (62\%). At the time of diagnosis, median number of HCC lesions was 2 (interquartile range [IQR], 1-3), and median size of the largest lesion was $2.5 \mathrm{~cm}$ (IQR, 1.75-3.9). Overall sensitivity of US study for detection of $\mathrm{HCC}$ was $33 \%$ (95\% confidence interval [Cl], 29-48\%). Sensitivity was $77 \%(95 \% \mathrm{Cl}, 62-93 \%)$ in patients with $\mathrm{BMI}<30$ and $21 \%(95 \% \mathrm{Cl}, 11-30 \%)$ in patients with $\mathrm{BMl} \geq 30(P<0.001)$. Size of the largest $\mathrm{HCC}$ lesion $(P=0.290)$ and number of lesions ( $P=0.505$ ) were not different between groups. Computed tomography (CT) scan detected HCC in $98 \%$ of the obese patients with negative US.

Conclusions: Sensitivity of US for detection of HCC is significantly lower among obese patients compared to overweight and normal weight patients. These patients may benefit from alternating between US and a different imaging modality. (Clin Mol Hepatol 2020;26:54-59)

Keywords: Liver cirrhosis; Neoplasms; Obesity

\footnotetext{
Study Highlights

The American Association for the Study of Liver Diseases (AASLD) recommends ultrasound (US) screening for hepatocellular carcinoma (HCC) among patients with cirrhosis, regardless of body mass index (BMI), every 6 months. However based on this study sensitivity of US for detection of $\mathrm{HCC}$ is significantly lower among obese patients compared to overweight and normal weight patients. These patients may benefit from alternating between US and a different imaging modality, i.e., computed tomography or magnetic resonance imaging.
}

\section{Abbreviations:}

AASLD, American Association for the Study of Liver Diseases; AFP, alfafetoprotein; $\mathrm{BMI}$, body mass index; $\mathrm{Cl}$, confidence interval; $\mathrm{CT}$, computed tomography; $\mathrm{HCC}$, hepatocellular carcinoma; IQR, interquartile range; MRI, magnetic resonance imaging; NAFLD, non-alcoholic fatty liver disease; NASH, non-alcoholic steatohepatitis; US, ultrasound

\author{
Corresponding author : Jamak Modaresi Esfeh \\ Department of Gastroenterology and Transplant Hepatology, Cleveland \\ Clinic, 9500 Euclid Avenue. Cleveland, OH 44195, USA \\ Tel: +1-216-501-2595, Fax: +1-216-445-3889 \\ E-mail:modarej@ccf.org \\ https://orcid.org/0000-0002-9429-5465
}




\section{INTRODUCTION}

Hepatocellular carcinoma (HCC) is the fourth leading cause of cancer-related death in the world. ${ }^{1}$ HCC usually happens in the context of pre-existing chronic liver disease, and its clinical diagnosis is difficult, commonly necessitating using an imaging modality for diagnosis. ${ }^{2}$ As cirrhosis is the single most important risk factor for HCC in North America, national and international clinical guidelines recommend surveillance of all patients with cirrhosis for $\mathrm{HCC}^{2-5}$ Imaging the liver or using blood levels of biomarkers have been studied as potential surveillance strategies. Measuring the plasma levels of alfa-fetoprotein (AFP) for screening of HCC has poor accuracy, ${ }^{6,7}$ and its use for surveillance, especially as a stand-alone test is discouraged by current practice guidelines. ${ }^{2}$ Although contrast-enhanced liver computed tomography (CT) or magnetic resonance imaging (MRI) have been validated as accurate diagnostic tests for HCC., ${ }^{8,9}$ their use for surveillance is costly and accumulating radiation exposure over time in case of $\mathrm{CT}$ is problematic. Therefore currently a liver ultrasound (US) every 6 months is recommended as the surveillance strategy of choice for patients with cirrhosis. ${ }^{2-4}$

Use of ultrasound for HCC surveillance in obese patients has been reported to be controversial due to potentially decreased accuracy. 5,10,11 Prevalence of obesity has been increasing both in United States and globally. ${ }^{12}$ Moreover, obesity is a major risk factor for non-alcoholic fatty liver disease (NAFLD) ${ }^{13-15}$ and recent studies show an increase in prevalence of NAFLD and non-alcoholic steatohepatitis (NASH) cirrhosis, as well as an increase in NAFLD as the underlying liver disease in patients with HCC. $^{16-20}$ Given these trends which lead to increasing frequency of obesity among patients with cirrhosis and $\mathrm{HCC}^{21,22}$ it is important to evaluate the impact of obesity on the accuracy of US for surveillance of HCC.

In this retrospective study, we evaluated the accuracy of US for surveillance of HCC among liver transplant patients, using pathologic examination of the explant as the gold standard for diagnosis of HCC. The effect of patients' body mass index (BMI) on the accuracy of US for HCC surveillance was assessed and compared to the results of MRI and $\mathrm{CT}$ in such population.

\section{MATERIALS AND METHODS}

\section{Study population and data collection}

The study protocol was approved by the Cleveland Clinic Insti- tutional Review Board. Between January 2012 and December 2017, consecutive liver transplant patients who were diagnosed with HCC based on the pathologic examination of their explant entered the study. In each explant, the number of HCC lesions, their diameter, and the histological differentiation of the tumor were recorded. For each liver transplant, patient's gender, age, $\mathrm{BMI}$ at the time of transplant, underlying liver disease, and date of transplant were recorded. Obesity was defined as BMI $\geq 30$. Electronic medical records of these patients were reviewed and data was extracted on the date of diagnosis of HCC. Also, the method of HCC diagnosis was recorded (i.e., US, CT, MRI, or diagnosed during the examination of the explant).

For patients whose HCC was first diagnosed based on a screening US, data was extracted from the US findings, including date of US, number of HCC lesions, and size of the largest lesion. For patients whose HCC was diagnosed based on a diagnostic test other than US, same data was extracted from a screening US within a 3-month period prior from the date of diagnosis of HCC. Similar data on presence or absence of $\mathrm{HCC}$ and characteristics of lesions were collected on CT and MRI findings when applicable.

Sensitivity of US for diagnosis of HCC was calculated and compared to the gold standard which was diagnosis of HCC in explant pathology. US sensitivity in diagnosis of HCC was compared between different BMI subgroups. Additionally, diagnostic utility of $\mathrm{CT}$ and MRI imaging was evaluated for diagnosis of HCC.

\section{Statistical analysis}

All analysis was done with stata data analysis and statistical software (version 11.2 SE; StataCorp LLC, College Station, TX, USA). Variables are reported as number (percentage), mean (standard deviation) or median (interquartile range, IQR). Categorical variables are compared between groups with chi-square test. Interval variables are compared between groups with Wilcoxon rank-sum and $t$-tests. Confidence intervals $(\mathrm{Cls})$ for proportions are calculated using normal distribution approximation. $P$-value less than $5 \%$ was defined as clinically significant. All $P$-values are two-sided.

\section{RESULTS}

\section{Patient characteristics}

Between January 2012 and December 2017, 169 consecutive 
liver transplantations were confirmed to have HCC in pathological examination of the explant. One hundred and sixteen patients had at least one screening US within the 3-month period of the date of diagnosis of HCC and were included in the study. Main risk factor for HCC in all these patients was known diagnosis of cirrhosis. AFP was elevated in $83 \%$ of the patients (mean of $48 \pm 12$ ). In all cases HCC was identified in the background of cirrhosis. On pathology exam, $66 \%$ of the patients had well-differentiated HCC, 30 had moderately-differentiated HCC, 4\% had poorly-differentiated HCC.

$73 \%$ of these patients received at least one type of locoregional therapy prior to liver transplant (including radiofrequency ablation/micro wave ablation in $41 \%$, transarterial chemoembolization and bland embolization in $25 \%$ and transarterial radioembloization in $7 \%$ of the patients). The majority of study population was male and obese ( $80 \%$ and $65 \%$, respectively). Mean BMI was $31 \mathrm{~kg} / \mathrm{m}^{2}$ (range, $20-43$ ). Overall $62 \%$ of the patients had a BMI $\geq 30 \mathrm{~kg} / \mathrm{m}^{2}$ and $38 \%$ of the patients had $\mathrm{BMI}<30 \mathrm{~kg} / \mathrm{m}^{2}$. The most common underlying liver disease was chronic hepatitis $\mathrm{C}$ virus infection in 72 patients (65.0\%), followed by alcoholic cirrhosis in $16(13.7 \%)$, and NASH in eight patients (6.8\%). Less common etiologies for cirrhosis were cryptogenic cirrhosis, chronic hepatitis B virus infection, primary biliary cholangitis, hemochro-

Table 1. Charactersitics of patients with HCC

\begin{tabular}{lc}
\hline Variable & Value \\
\hline Total & 116 \\
Male & $93(80)$ \\
Age, mean (range) & $58(48-71)$ \\
BMI & $31 \pm 4.4$ \\
BMI $\geq 30$ & $75(65)$ \\
\hline Hypertension & $42(7)$ \\
\hline Diabetes mellitus & $15(17)$ \\
MELD & $18 \pm 5$ \\
CTP score (\%) & \\
A & 13 \\
B & 34 \\
C & 53 \\
Number of HCC lesions* & $2(1-3)$ \\
Size of largest HCC lesion* (cm) & $2.5(1.75-3.9)$ \\
\hline
\end{tabular}

Values are presented as mean \pm standard deviation, number (\%), or median (interquartile range).

HCC, hepatocellular carcinoma; BMI, body mass index; MELD, Model for End-Stage Liver Disease; CTP, Child-Turcotte-Pugh.

*Based on pathological examination of the explant. matosis and alpha 1 antitrypsin deficiency, in order of frequency.

The mean Model for End-Stage Liver Disease Na score at the time of transplant was $18.13 \%$ of the patients were Child-Turcotte-Pugh class A, $34 \%$ class B and $53 \%$ class $C$ at the time of transplant. On average patients had $2.0(\mathrm{IQR}, 1-3) \mathrm{HCC}$ lesions on pathologic examination with the average size of the largest lesion being $2.5 \mathrm{~cm}$ (IQR, 1.75-3.9) (Table 1). In all patients, post transplantation immunosuppression regimen consisted of different combinations of tacrolimus, or cyclosporine, and mycophenolate mofetil and prednisone.

\section{Diagnosis of HCC and sensitivity of US}

Among the 116 patients, 38 (32.7\%) had their HCC diagnosed by US. Sixty-five $(56.0 \%)$ were diagnosed by $\mathrm{CT}$, three $(2.6 \%)$ with MRI, and $10(8.6 \%)$ were diagnosed after transplant by examination of the explant. The method of HCC diagnosis was significantly different depending on patient's body habitus and weight. While in the majority of patients (59\%) with $\mathrm{BMI}<30$,

Table 2. Characteristic of HCC lesions

\begin{tabular}{|c|c|c|c|}
\hline \multirow{2}{*}{$\begin{array}{l}\text { Characteristics of patients } \\
\text { and their HCC lesions }\end{array}$} & \multicolumn{2}{|c|}{ BMI } & \multirow{2}{*}{$P$-value } \\
\hline & $<30$ & $\geq 30$ & \\
\hline Number & $41(35)$ & $75(65)$ & NA \\
\hline Mean BMI & $26 \pm 2.5$ & $33 \pm 2.9$ & NA \\
\hline Mean age & $57 \pm 9$ & $59 \pm 7$ & 0.152 \\
\hline Male & $33(81)$ & $60(80)$ & 0.950 \\
\hline HCC first diagnosed by & & & $<0.001$ \\
\hline US & $24(59)$ & $14(19)$ & \\
\hline $\mathrm{CT}$ & $15(37)$ & $50(67)$ & \\
\hline MRI & $1(2)$ & $2(3)$ & \\
\hline Pathology & $1(2)$ & $9(12)$ & \\
\hline $\begin{array}{l}\text { Number of HCC lesions at } \\
\text { diagnosis* }\end{array}$ & $1(1-2)$ & $1(1-2)$ & 0.734 \\
\hline $\begin{array}{l}\text { Size of largest HCC lesion at } \\
\text { diagnosis* }(\mathrm{cm})\end{array}$ & $2.2(1.8-4)$ & $2(1.8-3)$ & 0.373 \\
\hline $\begin{array}{l}\text { Number of HCC lesions in } \\
\text { the explant }^{\dagger}\end{array}$ & $2(1-3)$ & $1(1-3)$ & 0.545 \\
\hline Sensitivity of US (CI) (\%) & $77(62-93)$ & $21(11-30)$ & $<0.001$ \\
\hline
\end{tabular}

Values are presented as mean \pm standard deviation, number (\%), or median (interquartile range) unless otherwise indicated.

HCC, hepatocellular carcinoma; BMI, body mass index; NA, not applicable; US, ultrasound; CT, computed tomography; MRI, magnetic resonance imaging.

${ }^{*}$ As reported at diagnosis with US, CT, MRI or pathological exam of explant. ${ }^{\dagger}$ As reported on pathological exam of the explant. 
the diagnosis was made with US; in the majority of patient $(67 \%)$ with $\mathrm{BMI} \geq 30$, HCC diagnosis was made with $\mathrm{CT}$ scan ( $P$-value $<0.001$ ) (Table 2).

Overall sensitivity of a single US for detection of HCC was $33 \%$ $(95 \% \mathrm{Cl}, 24-41 \%)$ in the study population. When sensitivity of US was calculated for each subgroup of patients based on their BMI, the sensitivity was $59 \%(95 \% \mathrm{Cl}, 43-74 \%)$ in non-obese patients, as compared to sensitivity of $19 \%(95 \% \mathrm{Cl}, 10-28 \%)$ in obese patients ( $P$-value $<0.001)$. Age, gender, number and size of largest HCC lesion at diagnosis were not significantly different between non-obese and obese patients (Table 2).

Seventeen non-obese patients (41\%) and 61 obese patients (81\%) had falsely negative US within 6 months of HCC diagnosis detected by another diagnostic method. Sixteen out of 17 nonobese patients (94\%) and 52 out of 61 obese patients (85\%) with falsely negative US had been evaluated for HCC with either CT or MRI. Alternate imaging with either CT or MRI had a sensitivity of $100 \%$ in both groups for diagnosis of HCC.

\section{DISCUSSION}

American Association for the Study of Liver Diseases (AASLD) recommends screening for $\mathrm{HCC}$ in cirrhotic patients using ultrasound every 6 months. ${ }^{23}$ The reported sensitivity of unenhanced ultrasound for detection of HCC varies significantly based on different studies (between 34\% and 100\%). ${ }^{24}$ However, it has been shown that sensitivity of ultrasound drops significantly in obese patients..$^{25}$ AASLD recommendations regarding HCC screening has been made regardless of the BMI of the patient. Considering the lower sensitivity of US in detecting HCC in obese patients, current HCC recommendations can potentially cause a delay in diagnosis of HCC in this patient population. This can be one of the reasons for the worse prognosis and larger tumor size in patients with NASH induced $\mathrm{HCC}^{25}$

This needs extra attention given the high prevalence and growing number of obese population in the United States. Based on the report form Centers for Disease Control and Prevention, the prevalence of obesity was $36.5 \%$ (crude estimate) among United States adult population during 2011-2014, which is more than one-third of this population. From 1999-2000 through 20132014 , a significant increase in obesity was observed in adults. ${ }^{26}$ Lower sensitivity of ultrasound in obese patients and risk of delay in timely diagnosis of HCC, has a significant effect on prognosis, treatment options (cure vs. palliative measures) as well as on long-term survival post liver transplant. Mazzaferro et al. ${ }^{27}$ showed a higher post transplantation recurrence rates in patients with single lesion larger than $5 \mathrm{~cm}$ or multiple nodules any of which exceeds $3 \mathrm{~cm}$. This is the rationale behind Milan criteria, used by United Network for Organ Sharing.

In a study done by Chalasani et al. ${ }^{28}$ in $1999,84 \%$ of the 473 members of AASLD indicated use of some form of surveillance practice, with majority (69\%) using ultrasound as the only imaging modality. In our study, ultrasound sensitivity in detecting HCC was assessed in the entire studied population as well as in different BMI sub-groups. Overall sensitivity of a single US for detection of HCC was $33 \%(95 \% \mathrm{Cl}, 24-41 \%)$ in the study population. It was shown that, sensitivity of ultrasound drops significantly as BMI increases. US sensitivity in detecting $\mathrm{HCC}$ was $59 \%(95 \% \mathrm{Cl}$, $43-74 \%$ ) in non-obese patients with $\mathrm{BMI}<30$, as compared to sensitivity of $19 \%(95 \% \mathrm{Cl}, 10-28 \%)$ in obese patients with $\mathrm{BM}$ $\geq 30$ ( $P$-value $<0.001)$.

This study has some limitations. First, this is a retrospective study with all its inherent limitations. Second, liver ultrasound was performed by different radiologists and therefore prone to interobserver variability. The other limitation of this study is possibility of missed sub centimeter HCCs on the explants which can be missed on 5-10 mm sectioning.

Theoretically, using an alternative method of imaging for $\mathrm{HCC}$ screening, CT scan or MRI, can potentially decrease the rate of missed cases of HCC and reduce the delay in diagnosis and treatment of this cancer. However, performing CT scan every 6 months (as recommended by AASLD), imposes a significant accumulative radiation dose over years. One option would be applying different methods of imaging, i.e., alternating ultrasound and $\mathrm{CT}$ scan or MRI every 6 months.

Based on this study, and due to the concern with US sensitivity in detecting $\mathrm{HCC}$-especially in obese patients- and given the prognostic significance of earlier diagnosis; a more sensitive imaging modality especially in obese population appears to be a necessity.

\section{Authors' contribution}

Jamak: Designed the study, Collected the data, wrote the paper Kaveh: Performed the analysis

Kianoush: Wrote the paper

\section{Conflicts of Interest}

The authors have no conflicts to disclose. 


\section{REFERENCES}

1. Global Burden of Disease Liver Cancer Collaboration, Akinyemiju T, Abera S, Ahmed M, Alam N, Alemayohu MA, et al. The burden of primary liver cancer and underlying etiologies from 1990 to 2015 at the global, regional, and national level: results from the global burden of disease study 2015. JAMA Oncol 2017;3:1683-1691.

2. Bruix J, Sherman M; American Association for the Study of Liver Diseases. Management of hepatocellular carcinoma: an update. Hepatology 2011;53:1020-1022.

3. European Association For The Study Of The Liver; European Organisation For Research And Treatment Of Cancer. EASL-EORTC clinical practice guidelines: management of hepatocellular carcinoma. J Hepatol 2012;56:908-943.

4. Ryder SD; British Society of Gastroenterology. Guidelines for the diagnosis and treatment of hepatocellular carcinoma (HCC) in adults. Gut 2003;52 Suppl 3:iii1-iii8.

5. Bruix J, Sherman M; Practice Guidelines Committee, American Association for the Study of Liver Diseases. Management of hepatocellular carcinoma. Hepatology 2005;42:1208-1236.

6. Singal $A$, Volk ML, Waljee A, Salgia $R$, Higgins $P$, Rogers $M A$, et al. Meta-analysis: surveillance with ultrasound for early-stage hepatocellular carcinoma in patients with cirrhosis. Aliment Pharmacol Ther 2009:30:37-47.

7. Lok AS, Sterling RK, Everhart JE, Wright EC, Hoefs JC, Di Bisceglie $A M$, et al. Des-gamma-carboxy prothrombin and alpha-fetoprotein as biomarkers for the early detection of hepatocellular carcinoma. Gastroenterology 2010;138:493-502.

8. Forner A, Vilana R, Ayuso C, Bianchi L, Solé M, Ayuso JR, et al. Diagnosis of hepatic nodules $20 \mathrm{~mm}$ or smaller in cirrhosis: prospective validation of the noninvasive diagnostic criteria for hepatocellular carcinoma. Hepatology 2008;47:97-104.

9. Sangiovanni A, Manini MA, lavarone $M$, Romeo $R$, Forzenigo LV, Fraquelli $M$, et al. The diagnostic and economic impact of contrast imaging techniques in the diagnosis of small hepatocellular carcinoma in cirrhosis. Gut 2010;59:638-644.

10. Mikami S, Tateishi R, Hagiwara S, Sato M, Minami T, Uchino K, et al. Tumor markers are more useful in patients undergoing surveillance for hepatocellular carcinoma with unreliable results by ultrasonography. Hepatol Res 2015;45:415-422.

11. Sato T, Tateishi R, Yoshida H, Ohki T, Masuzaki R, Imamura J, et al. Ultrasound surveillance for early detection of hepatocellular carcinoma among patients with chronic hepatitis C. Hepatol Int 2009;3:544-550.

12. Stevens GA, Singh GM, Lu Y, Danaei G, Lin JK, Finucane MM, et al. National, regional, and global trends in adult overweight and obesity prevalences. Popul Health Metr 2012;10:22.

13. Marchesini G, Bugianesi E, Forlani G, Cerrelli F, Lenzi M, Manini R, et al. Nonalcoholic fatty liver, steatohepatitis, and the metabolic syndrome. Hepatology 2003;37:917-923.

14. Wanless IR, Lentz JS. Fatty liver hepatitis (steatohepatitis) and obesity: an autopsy study with analysis of risk factors. Hepatology 1990;12:1106-1110.

15. Rinella ME. Nonalcoholic fatty liver disease: a systematic review. JAMA 2015;313:2263-2273.

16. Younossi ZM, Otgonsuren M, Henry L, Venkatesan C, Mishra A, Erario $M$, et al. Association of nonalcoholic fatty liver disease (NAFLD) with hepatocellular carcinoma (HCC) in the United States from 2004 to 2009. Hepatology 2015;62:1723-1730.

17. Hashimoto $E$, Tokushige K. Hepatocellular carcinoma in non-alcoholic steatohepatitis: growing evidence of an epidemic? Hepatol Res 2012;42:1-14.

18. Bugianesi E, Leone N, Vanni E, Marchesini G, Brunello F, Carucci P, et al. Expanding the natural history of nonalcoholic steatohepatitis: from cryptogenic cirrhosis to hepatocellular carcinoma. Gastroenterology 2002;123:134-140.

19. Hashimoto E, Yatsuji S, Tobari M, Taniai M, Torii N, Tokushige $K$, et al. Hepatocellular carcinoma in patients with nonalcoholic steatohepatitis. J Gastroenterol 2009;44 Suppl 19:89-95.

20. Ascha MS, Hanouneh IA, Lopez R, Tamimi TA, Feldstein AF, Zein NN. The incidence and risk factors of hepatocellular carcinoma in patients with nonalcoholic steatohepatitis. Hepatology 2010;51:19721978.

21. Yasui K, Hashimoto E, Komorizono Y, Koike K, Arii S, Imai Y, et al. Characteristics of patients with nonalcoholic steatohepatitis who develop hepatocellular carcinoma. Clin Gastroenterol Hepatol 2011;9:428-433.

22. Mittal S, El-Serag HB, Sada YH, Kanwal F, Duan Z, Temple S, et al. hepatocellular carcinoma in the absence of cirrhosis in United States veterans is associated with nonalcoholic fatty liver disease. Clin Gastroenterol Hepatol 2016;14:124-131.e1.

23. Heimbach JK, Kulik LM, Finn RS, Sirlin CB, Abecassis MM, Roberts $L R$, et al. AASLD guidelines for the treatment of hepatocellular carcinoma. Hepatology 2018;67:358-380.

24. Yu NC, Chaudhari V, Raman SS, Lassman C, Tong MJ, Busuttil RW, et al. CT and MRI improve detection of hepatocellular carcinoma, compared with ultrasound alone, in patients with cirrhosis. Clin Gastroenterol Hepatol 2011;9:161-167.

25. Kolly P, Dufour JF. Surveillance for hepatocellular carcinoma in patients with NASH. Diagnostics (Basel) 2016;6:22.

26. Hales CM, Carroll MD, Fryar CD, Ogden CL. Prevalence of obesity among adults and youth: United States, 2015-2016. NCHS Data Brief 2017;(288):1-8.

27. Mazzaferro V, Regalia E, Doci R, Andreola S, Pulvirenti A, Bozzetti F, et al. Liver transplantation for the treatment of small hepatocellular carcinomas in patients with cirrhosis. N Engl J Med 1996;334:693-699. 
Jamak Modaresi Esfeh, et al.

Ultrasound sensitivity in liver cancer

28. Chalasani N, Said A, Ness R, Hoen H, Lumeng L. Screening for hepatocellular carcinoma in patients with cirrhosis in the United States: results of a national survey. Am J Gastroenterol 1999;94:22242229. 